\title{
Combined transarterial chemoembolization and stereotactic body radiation therapy as a bridge therapy to liver transplant for hepatocellular carcinoma
}

\author{
Bui Quang Bieu${ }^{1}$, Nguyen Dinh Chau ${ }^{1}$, Nguyen Xuan Kien¹, Le Van Thanh², Vu Van Quang ${ }^{2}$, Thai Doan Ky ${ }^{3}$, \\ Nguyen Tien Thinh ${ }^{3}$, Mai Hong Bang ${ }^{3}$ \\ 'Department of Radiation Oncology and Radiosurgery, Military Central Hospital 108, Hanoi 100000, Vietnam. \\ 2Department of Hepatobiliary and Pancreatic Surgery, Military Central Hospital 108, Hanoi 100000, Vietnam. \\ ${ }^{3}$ Department of Gastroenterology and Hepatobiliary, Military Central Hospital 108, Hanoi 100000, Vietnam.
}

Correspondence to: Dr. Bui Quang Bieu, Department of Radiation Oncology and Radiosurgery, Military Central Hospital 108, Hanoi 100000, Vietnam. E-mail: buiquangbieu@gmail.com

\begin{abstract}
How to cite this article: Bieu BQ, Chau ND, Kien NX, Thanh LV, Quang VV, Ky TD, Thinh NT, Bang MH. Combined transarterial chemoembolization and stereotactic body radiation therapy as a bridge therapy to liver transplant for hepatocellular carcinoma. Hepatoma Res 2020;6:49. http://dx.doi.org/10.20517/2394-5079.2020.39
\end{abstract}

Received: 17 Apr 2020 First Decision: 18 May 2020 Revised: 19 Jun 2020 Accepted: 14 Jul 2020 Published: 15 Aug 2020

Academic Editors: Su Pin Choo, Guang-Wen Cao, David Tan Boon Harn Copy Editor: Cai-Hong Wang Production Editor: Jing Yu

\begin{abstract}
Liver transplant (LT) is the curative treatment for patients with hepatocellular carcinoma (HCC). Bridge therapies are local treatments given to patients on the LT waitlist, to prevent tumor progression and to reduce the dropout rate. Case presentation: We reported a 40-year-old man diagnosed with Barcenola-Clinic Liver Cancer BCLC intermediate stage $\mathrm{HCC}$ and Child-Pugh A5 hepatitis B virus cirrhosis who underwent combined bridge therapies to LT. Firstly, the patient received transarterial chemoembolization (TACE) for two times and showed a partial response. Then he underwent stereotactic body radiation therapy (SBRT) with a total dose of 45 Gy in 3 fractions. Three months later, the tumor size and serum protein induced by Vitamin $\mathrm{K}$ absence or antagonists-II, alpha fetoprotein levels decreased gradually. In June 2019 a suitable donor was found and his LT was successfully performed. Conclusion: We propose that a combination of TACE and SBRT was feasible as bridge therapy for HCC patients on the LT waitlist.
\end{abstract}

Keywords: Transarterial chemoembolization, stereotactic body radiation therapy, bridge therapy, hepatocellular carcinoma, liver transplant

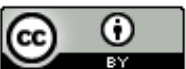

(C) The Author(s) 2020. Open Access This article is licensed under a Creative Commons Attribution 4.0 International License (https://creativecommons.org/licenses/by/4.0/), which permits unrestricted use sharing, adaptation, distribution and reproduction in any medium or format, for any purpose, even commercially, as long as you give appropriate credit to the original author(s) and the source, provide a link to the Creative Commons license, and indicate if changes were made.

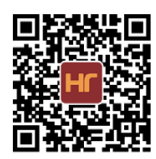




\section{INTRODUCTION}

Hepatocellular carcinoma (HCC) is one of the 5 most common cancers worldwide, and its incidence is increasing in Vietnam and Southeast Asian countries ${ }^{[1]}$. According to Milan criteria, the liver transplant (LT) is the treatment of choice for HCC patients with tumor less than $5 \mathrm{~cm}$ and up to 3 tumors $\leq 3 \mathrm{~cm}$. The 5 years survival rate in these patients are $70 \%$ with less than $20 \%$ recurrence rate ${ }^{[2]}$. However, not all HCC patients can undergo transplantation due to a lack of liver donors, resulting in an extended time on the waiting list $(\mathrm{WL})$ and a high dropout rate ${ }^{[3]}$.

Bridging treatments are locoregional therapies given to HCC patients on the WL to reduce the disease progression as well as the dropout rate. These treatments act as a temporary "bridge" until a suitable donor is identified. Liver resection, radiofrequency ablation (RFA), transarterial chemoembolization (TACE), and stereotactic body radiation therapy (SBRT) are main bridge modalities to LT in patients with HCC ${ }^{[2]}$. The rates of drop out at 6 months and 1 year were estimated as high as $12 \%$ and $15 \%-30 \%$, respectively, if HCC is left untreated ${ }^{[4,5]}$. The strongest risk factors for dropout from WL are tumor size $\geq 3 \mathrm{~cm}$ or multiple tumors, waiting time $\geq 6$ months, alpha fetoprotein (AFP) $\geq 200 \mathrm{ng} / \mathrm{mL}$, and poor response to bridging therapies ${ }^{[6]}$. In patients with HCC within Milan criteria, bridging therapy is estimated to decrease the dropout rate to $0-10 \%$. To reduce the dropout rate from the WL, a consensus statement recommends that bridging therapies should be considered for HCC patients with one nodule size $2 \mathrm{~cm}-5 \mathrm{~cm}$ or up to 3 nodules each $\leq 3 \mathrm{~cm}$, expected to wait longer than 6 months ${ }^{[2]}$.

Another aim of bridge therapy is to treat patients initially outside criteria for LT to fulfill Milan criteria which allows entry to the WL for LT after an adequate period of follow-up. In this case, bridge therapy is used as a downstaging procedure ${ }^{[3]}$.

There are several studies of bridge therapy to LT for HCC with $\mathrm{TACE}^{[7-9]}$ or $\mathrm{SBRT}^{[10-12]}$ alone. Some trials have shown that adjuvant SBRT post-TACE was safe and effective for patients with $\mathrm{HCC}^{[13-15]}$. In this paper, we report an HCC patient in the BCLC intermediate stage who we successfully treated with a combination of TACE and SBRT as a bridge therapy to LT in our center.

\section{CASE REPORT}

A 39-year-old male with a history chronic hepatitis B virus infection was diagnosed with intermediate BCLC HCC in October 2018. His liver function test (LFT) showed that he had Child-Pugh A5 cirrhosis. The tumor was in VI-VII segments and the patient characteristics were shown in Table 1. He had one tumor with a size bigger than $5 \mathrm{~cm}$ and very high serum AFP levels.

Because the patient was young with a good performance status of ECOG 0 , the LT team decided that he was an optimal candidate for LT and bridge therapy was needed to downstage the tumor while he was on the WL. Two DC beads TACE treatments were carried out in October and November 2018. The DC beads TACE technique was done in the same manner as conventional TACE. We used doxorubicin (Ebewe, Austria) loaded with DC-beads (Bicompatibles, UK) at least 90-120 min before the intervention. The dose of doxorubicin was $100 \mathrm{mg}$ per each treatment. Two sizes of DC-beads were used (100-300 $\mu \mathrm{m}$ and 300-500 $\mu \mathrm{m})$. These treatments resulted in partial response according to the modified Response Evaluation Criteria in Solid Tumors and Response Evaluation Criteria in Cancer of the Liver ${ }^{[16]}$.

Due to serum AFP level still too high post-TACE, as well as there was no suitable donor, we decided that he needs further bridge therapy with SBRT. The patient underwent SBRT with a total dose of 45 Gy in 3 fractions, one fraction delivered in every other day in January 2019. 
Table 1. Patient characteristics pre-treatment and post-TACE and SBRT

\begin{tabular}{llcc}
\hline Lab test and imaging & Pre-treatment & Post-TACE 1 months & Post-SBRT 3 months \\
\hline AFP $(I U / m L)$ & 2479 & 1128 & 143 \\
PIVKA-II $(\mathrm{mAU} / \mathrm{mL})$ & 272.6 & 29.4 & 0.8 \\
Child Pugh & $\mathrm{A} 5$ & $\mathrm{~A}$ & $\mathrm{~A} 5$ \\
AST & 28 & 43 & 47 \\
ALT & 33 & 65 & 70 \\
Tumor size $(\mathrm{mm})$ & $71 \times 60 \times 53$ & $72 \times 63 \times 43$ & $60 \times 43 \times 38$ \\
\hline
\end{tabular}

PIVKA: a protein induced by vitamin K absence; AST: aspartate transaminase; ALT: alanine transaminase; TACE: transarterial chemoembolization; SBRT: stereotactic body radiation therapy; AFP: alpha fetoprotein

\section{SBRT procedure for the patient}

CT simulation in GE CT 580 RT (USA)

The patient lied supine, arm up, and was immobilized in a vacuum bag (Qfix - USA). A non-contrast 4D CT performed with $2.5 \mathrm{~mm}$ slice thickness (used for treatment planning). A contrast-enhanced 4D CT with $2.5 \mathrm{~mm}$ slice thickness, intravenous injection of Omnipaque (GE Healthcare) $2 \mathrm{~mL} / \mathrm{kg}$, and $2.5 \mathrm{~mL} / \mathrm{second}$ was performed (used for target volume contouring). Both $4 \mathrm{D}$ CT data sets were then transferred to the treatment planning system (TPS).

Target volume and organs at risk contouring using Eclipse 13.6 (Varian, USA)

The average CT images were created for both non-contrast and contrast-enhanced 4D CT data sets. Noncontrast average CT was co-registered and fused with the contrast-enhanced one. Then 10 gross tumor volumes (GTVs) were contoured in 10 contrast-enhanced 4D CT data sets: only delineated tumor with contrast enhancement. The 10 GTVs were copied to contrast-enhanced average CT data set and combined to create internal target volume (ITV). The ITV was propagated from contrast-enhanced average CT data set to the non-contrast one. The planning target volume (PTV) was created in non-contrast average CT from ITV: PTV $=$ ITV $+5 \mathrm{~mm}$. The organs at risk were contoured in non-contrast average CT including liver, lung, heart, stomach, duodenum, small and large bowel, spinal cord, chest wall, kidneys, and gall bladder.

\section{Dose prescription}

The prescription dose was 45 Gy in 3 fractions, based on normal tissue constraints (the report of AAPM Task Group $\left.101^{[17]}\right)$.

\section{Treatment planning}

Treatment technique was VMAT with 2 coplanar arcs. Plan optimization was done in Eclipse 13.6 (Varian, USA). Based on normal tissue constraints, we selected a treatment plan with a total dose of 45 Gy in 3 fractions for the patient [Figure 1].

SBRT treatment plan parameters were shown in Table 2, with plan normalization 100\% prescription dose covered 95\% PTV and doses of OARs were within tolerance of AAPM Task Group 101 ${ }^{[16]}$. Normal liver volume received less than $17 \mathrm{~Gy}(\mathrm{~V} 17)$ was $812.7 \mathrm{~mL}$.

\section{Quality assurance of treatment plan}

The treatment plan was verified by portal dose dosimetry (Varian, USA) with a $2 \% / 1 \mathrm{~mm}$ gamma passing rate of $99.5 \%$.

\section{Treatment delivery}

The patient was treated in TrueBeam STx (Varian, USA) with position and immobilization just like in CT simulation. We used the Optical Surface Monitoring System (OSMS - Vision RT, UK) to help set up and 
45Gy/3Fx - Treatment Approved - Transversal - CT_RP_Ave (Avg)

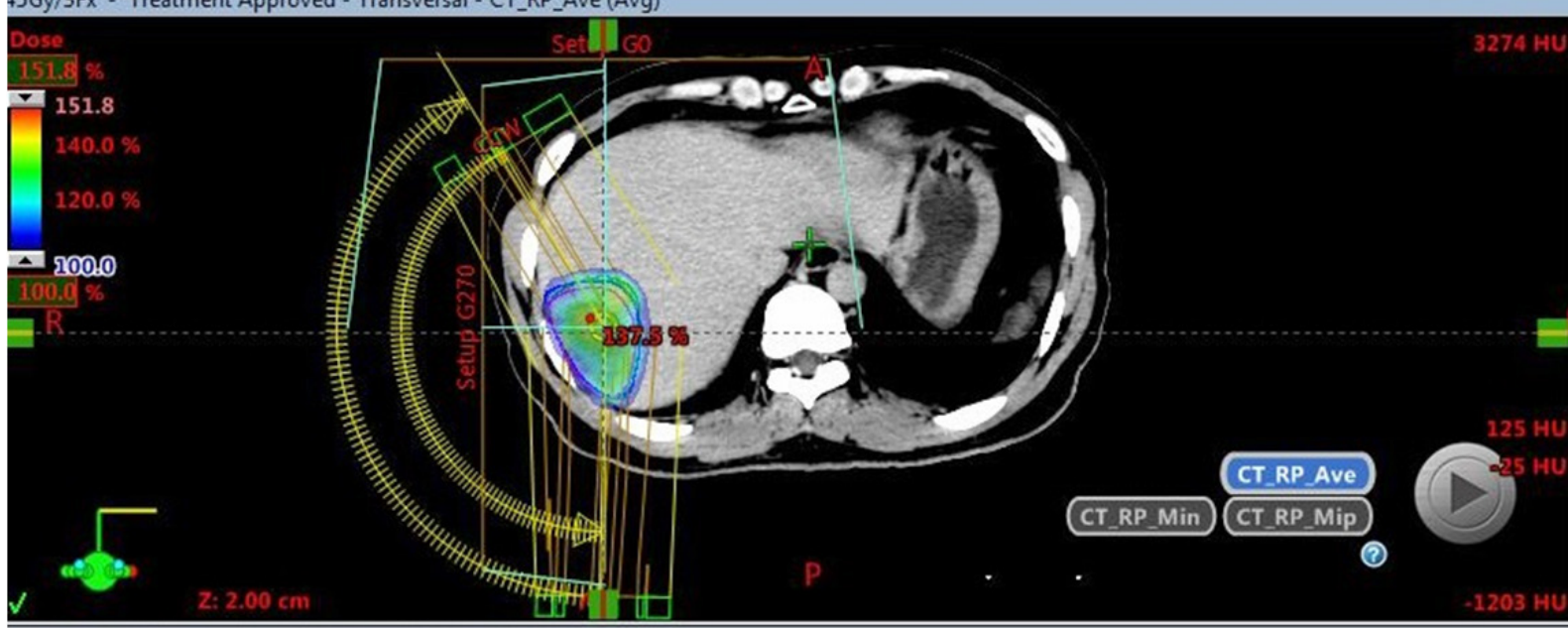

45Gy/3Fx - Treatment Approved - Frontal - CT_RP_Ave (Avg)

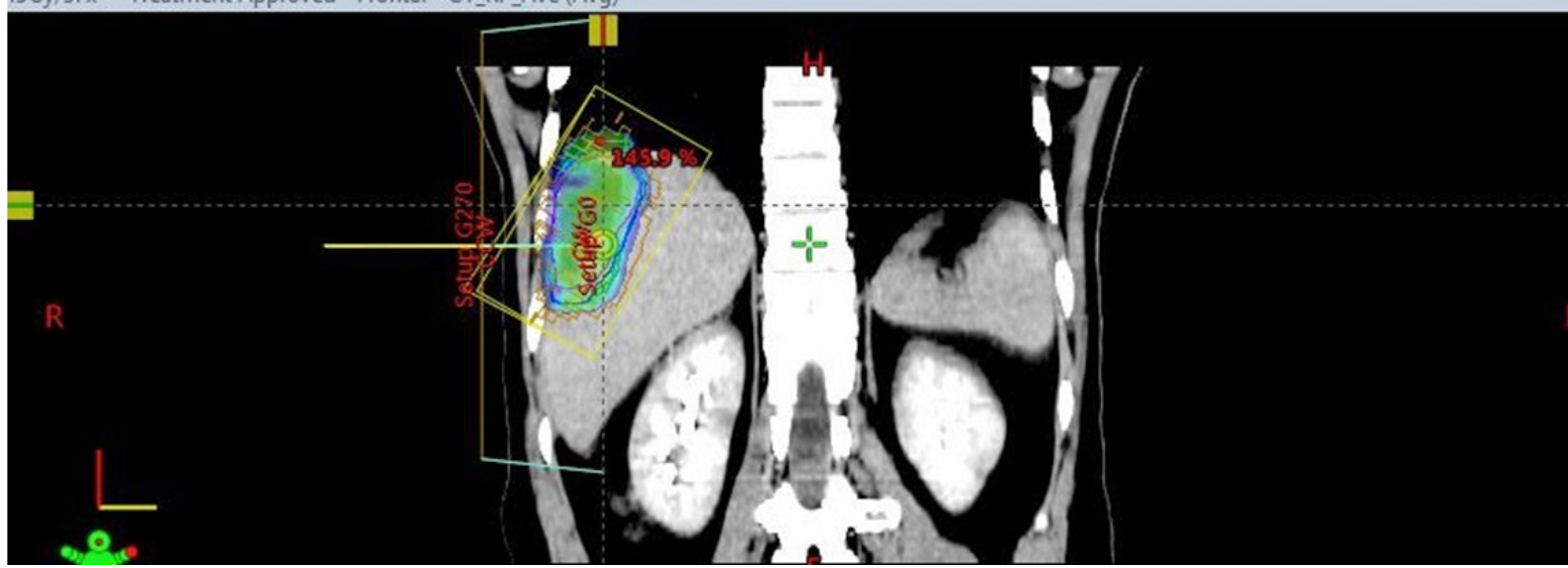

Figure 1. SBRT treatment plan and 100\% dose color wash (with courtesy of Department of Radiation Oncology and Radiosurgery, Military Central Hospital 108). SBRT: stereotactic body radiation therapy

Table 2. SBRT treatment plan parameters

\begin{tabular}{lcccc}
\hline Structures & Volume $\left.\mathbf{( c m}^{\mathbf{3}}\right)$ & Min dose $\mathbf{( G y )}$ & Max dose (Gy) & Mean dose (Gy) \\
\hline ITV & 130 & 43.97 & 68.31 & 55.62 \\
PTV & 201.3 & 34.68 & 68.31 & 53.44 \\
Liver & 1235.1 & 0.26 & 66.46 & 17.11 \\
Normal liver & 1112.5 & 0.26 & 65.93 & 12.87 \\
Gallbladder & 28.9 & 3.03 & 24.42 & 12.43 \\
Duodenum & 40 & 0.26 & 8.08 & 2.3 \\
Stomach & 265.5 & 0.26 & 5.4 & 2.3 \\
Jejunum & 327.8 & 0.08 & 2.8 & 0.5 \\
Ileum & 769.3 & 0.07 & 14.62 & 1.14 \\
Esophagus & 12 & 3.43 & 8.54 & 5.65 \\
Lung & 888.9 & 0.13 & 65.98 & 3.81 \\
Heart & 359.3 & 0.19 & 8.9 & 2.16 \\
Spinal cord & 35.9 & 0.03 & 7.48 & 2.08 \\
Right kidney & 129.2 & 0.14 & 2.02 & 0.53 \\
Left kidney & 134.3 & 0.05 & 0.48 & 0.17 \\
\hline
\end{tabular}

SBRT: stereotactic body radiation therapy; ITV: internal target volume; PTV: planning target volume

monitor patient movement during treatment. The patient's position was verified by cone-beam CT before each fraction. After the verification was done, the linac was beamed on to treat the patient. Total treatment 
time for each fraction was about $30 \mathrm{~min}$ : patient set up and verification $20 \mathrm{~min}$, beam on $10 \mathrm{~min}$. The total SBRT course lasted for two weeks.

\section{Follow-up}

The patient was treated with tenofovir (Savi Tenofovir) $300 \mathrm{mg}$ one tablet per day and scheduled for checkup one month post-SBRT and then every 3 months. Clinical examination, lab tests, and imaging were done during checkup including cell blood count, LFT, serum AFP, PIVKA II, abdominal ultrasound, chest Xray and abdominal CT.

The patient was well tolerated with SBRT and showed only minimum adverse effects such as mild fever and increased AST, ALT. Three months post SBRT, his LFT was still Child Pugh A5, serum PIVKA II and AFP levels were decreased, the tumor size was also decreased with central necrosis [Table 1, Figure 2]. In July 2019, we found a suitable donor for him, and his LT was successfully performed in August 2019. His post LT histopathology report showed that the tumor was mostly necrotized. He went on with tenofovir (Savi Tenofovir) $300 \mathrm{mg}$ one tablet per day, tacrolimus (Prograf) $1 \mathrm{mg}$ six capsules per day to treat HBV, and prevent rejection and routine follow-up. Until now, seven months post LT, he has no evidence of HCC, his LFT is normal with a mild increase of AST, ALT.

\section{DISCUSSION}

Several locoregional therapies have been used as bridging treatments for HCC patients awaiting LT. The most common treatments include TACE, RFA, and recently SBRT. Nowadays, TACE is still the most widely used as bridging therapy. In the procedure, a chemotherapeutic drug (commonly doxorubicin, cisplatin or mitomycin C), emulsified in lipiodol with embolizing material, is injected into the hepatic artery branch that feeding the tumor, to induce hypoxemia and tumor necrosis. The technique has been enhanced by drug-eluting beads (DEB-TACE), which allows a higher dose and uptake of chemotherapeutic drugs into the tumor and less systemic toxicity. In the histological examination, TACE achieves a complete pathological response in less than $30 \%$ of cases. Some studies focused on the efficacy of TACE as bridging treatment to LT on dropout rates in WL, survival, and recurrence after LT. The reported results of bridge therapy with TACE are controversial and no prospective randomized control trials have confirmed its efficacy in reducing dropout rates ${ }^{[2]}$. Several authors demonstrated that a good response to TACE (necrosis $>60 \%$ ) is significantly related to improved long-term survival after LT and a lower recurrence rate ${ }^{[18]}$. Others did not find any significant advantage of bridge therapy with TACE in overall and recurrence-free survival after LT in HCC patients ${ }^{[19,20]}$.

SBRT uses stereotactic conformal RT with 1-5 fractions of large fraction sizes (8-20 Gy/fraction) to the tumor while reducing the dose to adjacent normal tissues. The precise treatment and steep dose gradient within the target volume lead to excellent conformity with steep dose fall-off and high dose delivery to the target volume ${ }^{[21]}$. These advantages of SBRT over conventional radiotherapy allows a high chance of tumor control and minimizing treatment toxicities. SBRT for liver tumors was first introduced in the $1990 \mathrm{~s}^{[22]}$; however, it has not frequently been performed because of the concern of radiation-induced liver disease (RILD). Recently, with the development of medical linear accelerators and motion management solutions as well as supported data, SBRT has been recommended as a local treatment for HCC by NCCN guidelines. Now it is considered as an option for HCC patients who are not candidates to other bridging therapies ${ }^{[23]}$.

Data regarding the use of SBRT as a bridging treatment are emerging. In a paper by Sandroussi et al. ${ }^{[24]}$, ten HCC patients awaiting LT with tumor diameters ranging from 2.5 to $10.8 \mathrm{~cm}$ received conformal radiation therapy in 5-6 fractions. The treatment was done in nine patients with acceptable toxicities. Five patients underwent LT, and their explant pathology report showed that tumor necrosis ranging from 40\%-90\%. At a median follow-up of 6 months, no patients had tumor recurrence after LT. The author suggested that 
A
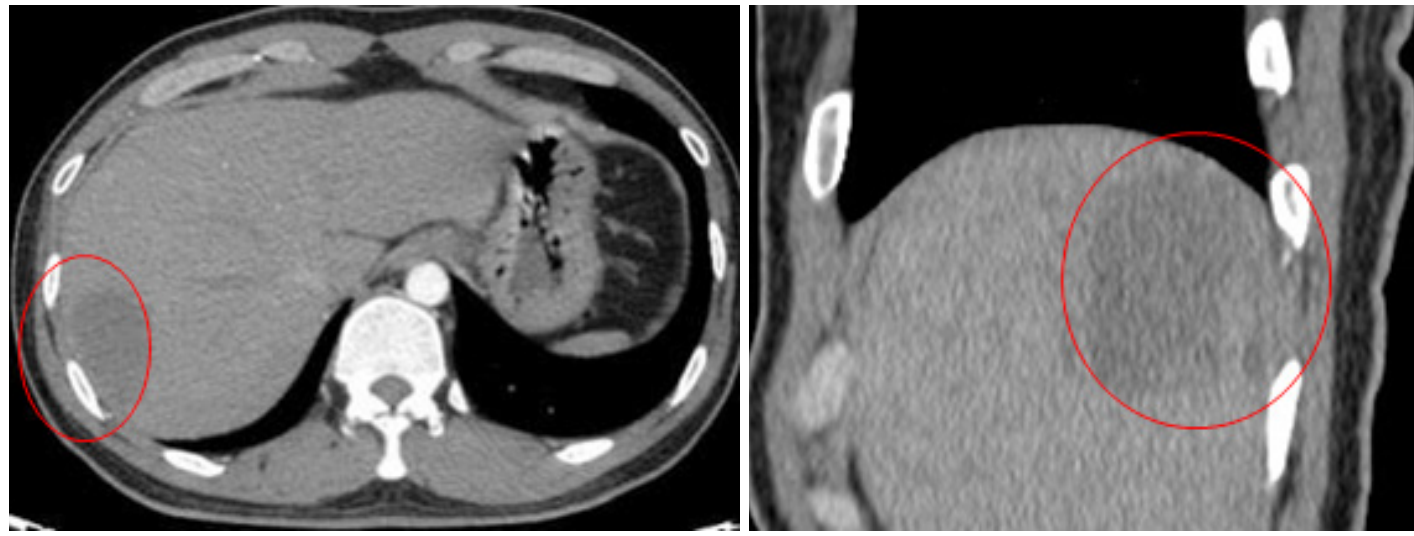

B
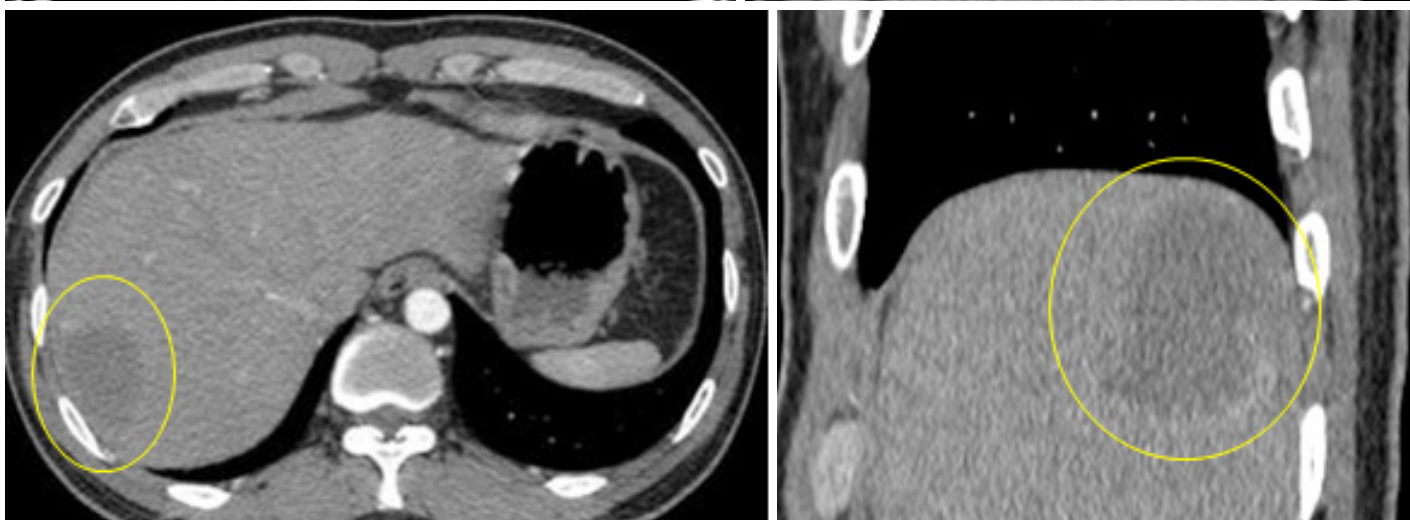

C
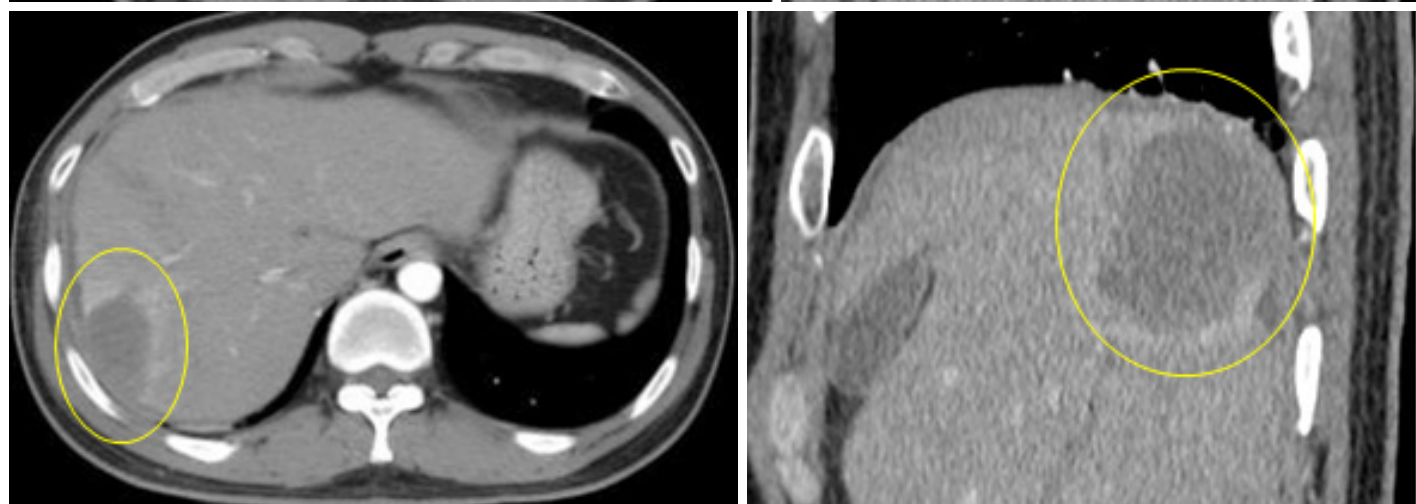

D
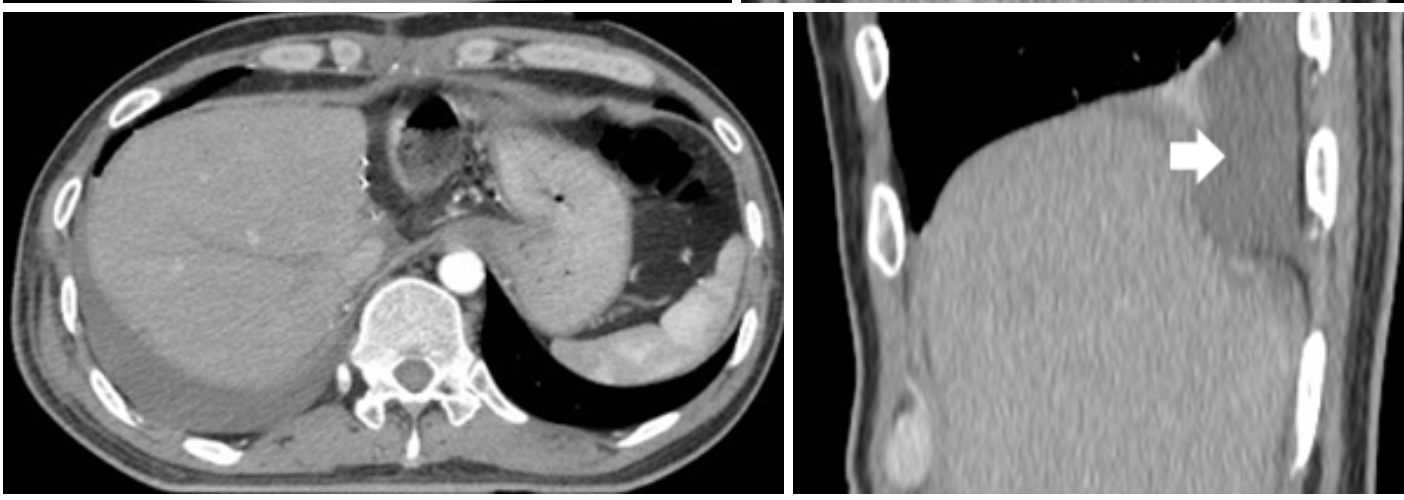

Figure 2. The patient's $C T$ images. A: non-contrast enhancing primary tumor (red rings) in arterial phage CT images pre SBRT; $B, C$ : mild contrast-enhancing of normal liver tissue surrounding the primary tumor (yellow rings) in arterial phage CT images one month and three months post-SBRT; D: new transplanted liver and local pleural effusion in the right lung (white arrow). With courtesy of Department of Radiation Oncology and Radiosurgery and Department of Hepatobiliary and Pancreatic Surgery, Military Central Hospital 108. SBRT: stereotactic body radiation therapy 
conformal radiation therapy is a safe and efficacious local bridging therapy for patients with HCC on the WL for $\mathrm{LT}^{[24]}$. In another article, Sapisochin et al.$^{[12]}$ reported an intention to treat analysis about SBRT used as bridge therapy in HCC patients not eligible for other locoregional treatments and observed similar dropout rate with SBRT and TACE or RFA. SBRT is proven to be safe and effective for tumors with a diameter $<6 \mathrm{~cm}$, even in lesions near the central biliary system, where surgery or RFA is impossible ${ }^{[25]}$. In a recent study by Moore et al ${ }^{[26]}, 23$ early-stage HCC patients who were not candidates for resection or local therapy treated with SBRT as bridge therapy to LT. The median prescribed doses to the tumor and the normal livers were 54 Gy (range 30-54 Gy) and 6.0 Gy (range 1.6-12.6 Gy), respectively. 22 patients had no significant changes in lab tests in 12 weeks follow-up but one patient developed RILD. 16 patients were on WL post SBRT and 11 were successfully transplanted. The median overall survival (OS) and progression-free survival (PFS) for the transplanted patients were not reached (range, 2.0-53.7 months, and 54 months, respectively) and were 23 and 14 months, respectively for the non-transplanted patients. Pathology report of liver explant post LT revealed 3 tumors (27.3\%) with complete response (CR), 6 tumors (54.5\%) with partial response (PR), and stable disease in 2 tumors (18.2\%). The authors concluded that SBRT was effective and safe to be used as a bridge therapy to LT without compromising the surgical procedure ${ }^{[26]}$. Furthermore, in a retrospective study, Gresswell et al. ${ }^{[27]}$ found that SBRT with functional treatment planning can be used safely as a bridge to LT in select patients with $\mathrm{CP} \geq 8$ cirrhosis.

Until now, there are no guidelines available to define which bridging therapy is the preferred treatment for specific patients. The choice of suitable bridging therapies has to be tailored to the the patient's status, the tumor characteristics, and more important the center experience. RFA is the treatment of choice in patients with a single tumor size $<5 \mathrm{~cm}$. The benefit of RFA as bridging therapy is best seen in patients with small tumors $<3 \mathrm{~cm}$ and $<1$-year waiting time ${ }^{[28]}$. TACE should be considered for patients with HCC between $3-5 \mathrm{~cm}$, because nodules with $3 \mathrm{~cm}$ of diameter or more are better vascularized, with a large feeding artery, therefore the effectiveness of TACE appears to be better; whereas smaller HCC has not yet a completely developed arterial neoangiogenesis ${ }^{[2,30]}$. SBRT has the advantage to treat the tumors adjacent to the central biliary system, in the liver dome or subcapsular HCC, these lesions are not suitable for RFA. However, SBRT is not suitable for tumors close to the duodenum, stomach, or bowel, for high risk of ulcer, hemorrhage, and perforation ${ }^{[25]}$. Up to now, there are several ongoing prospective phases 2 and 3 randomized trials to compare the safety and effectiveness of SBRT and TACE as a bridge therapy to $\mathrm{LT}^{[31,32]}$.

Experiences with combined therapies such as SBRT and TACE have been published in recent years, mostly in the scenario of unresectable HCC with diameter $>3 \mathrm{~cm}^{[33,34]}$. The rationale for treatment combination is to achieve a higher local control rate due to higher rates of complete tumor necrosis. The potential advantages when combining TACE followed by SBRT are: (1) TACE is most effective at the center of the HCC and failures are most commonly seen at the periphery of the tumor, where the ischaemic effects of TACE are least potent because the surrounding normal liver parenchyma is well oxygenated; (2) on the contrary, SBRT is most effective in the well-oxygenated periphery of the HCC and failures often occur in the more hypoxic zone at the tumor center; (3) large tumors that are not suitable for SBRT alone become more amenable to this therapy following TACE due to the effect of TACE in the hypoxic area at the tumor center; and (4) a theoretical radio-sensitization by the cytotoxic agents used in TACE may result in the improvement of tumor response ${ }^{[34]}$.

The approach of SBRT and TACE combination may be applied even as bridging therapy to LT. A retrospective study combined TACE followed by SBRT in patients with HCC size $\geq 3 \mathrm{~cm}$ by Jacob et al. ${ }^{[33]}$ showed that local recurrence was significantly decreased in the TACE plus SBRT group (10.8\%) when compared with the TACE-only group $(25.8 \%)(P=0.04)$. The TACE plus SBRT group also had significantly longer OS than the TACE-only one (33 months and 20 months, respectively; $P=0.02$ ). The author supposed that combined TACE and SBRT resulted in a survival advantage over treatment with TACE 
alone in HCC patients with tumor size $\geq 3 \mathrm{~cm}$ and a prospective randomized clinical trial is required to confirm the result ${ }^{[33]}$. In another pilot phase II trial, Buckstein et al. ${ }^{[34]}$ combined drug-eluting bead (DEB) TACE followed by SBRT in 25 HCC patients with single tumor size $4-7 \mathrm{~cm} .92 \%$ of target lesions showed objective response including $64 \% \mathrm{CR}(n=16)$ and $28 \%$ PR $(n=7) .2$-year OS and PFS were $67 \%$ and $52 \%$, respectively. Cause-specific survival (CSS) was $91 \%$ at 1 year and $83 \%$ at 2 years. He suggested that the results show very promising response rates when combining TACE and SBRT in large, unresectable HCC with the excellent OS, PFS, and $\operatorname{CSS}^{[34]}$. However, up to date, we do not found any paper address the combination of TACE and SBRT as a bridge therapy to LT for patients with HCC.

In our case, the patient had a big tumor size $>5 \mathrm{~cm}$ with very high serum PIVKA II and AFP levels so we combined both TACE and SBRT to increase the chance of local control as well as downstage the lesion to LT criteria. TACE was performed first and adjuvant SBRT was followed to exploit the advantage of combination therapies. It took 6 months for both therapies to downstage the tumor and 10 months on WL for the patient to find a suitable donor. After two TACE treatments, the tumor showed partial response but the serum AFP was still higher than $200 \mathrm{ng} / \mathrm{mL}$. The patient had a high risk of drop out from the WL for LT post TACE because there was still no suitable donor for him. Adding SBRT had kept the patient in the WL and finally, his LT was successfully done.

However, SBRT for HCC is a highly specialized procedure requiring both clinical and technical expertise. Considerations such as accurate target localization, rigid patient immobilisation, strict motion management, and proximity to adjacent viscera such as bowel and biliary structures must be considered prior to, and throughout treatment. As such, this technique can only be delivered safely and effectively in experienced centers with synchronized equipment, coupled with a well-oiled multi-disciplinary team comprising radiation oncologists, medical physicists and radiation therapy technologists.

At our center, we use $4 \mathrm{D} \mathrm{CT}$, gating, breath-hold techniques, or abdominal compression to manage the respiratory movement of the tumor, so that we can treat the tumor precisely with a 3-5 $\mathrm{mm}$ margin from ITV to PTV. Moreover, when combining SBRT with TACE as a bridge therapy to LT, it is necessary to have close teamwork between surgeons, gastroenterologists, and radiation oncologists. Additionally, adjuvant SBRT to TACE can do more harm to liver function and cause gastrointestinal toxicities such as bleeding or ulcer. So patient selection is very important to safely combine TACE and SBRT. Our patient had a good liver function with CP A5 and normal liver volume $>700 \mathrm{~mL}$. His tumor was close to the chest wall and liver dome so it is difficult for RFA and more suitable for adjuvant SBRT post-TACE. That is why he had only grade hepatic toxicity and no gastrointestinal toxicities post-TACE and SBRT. It is also important that after bridge therapy with TACE and SBRT, patients must continue the treatment of chronic liver disease, in our case was HBV, to prevent tumors from progression.

Finally, we should be cautious when evaluating treament response after TACE combined with SBRT for HCC. With this patient, we found that the tumor size did not change one month after SBRT and only decreased slightly 3-month post-SBRT with central necrosis. Interestingly, contrast enhancement was seen in normal liver tissue surrounding the primary tumor one month post SBRT and it seemed increased with time [Figure $2 \mathrm{~B}$ and $\mathrm{C}$ ]. It was a normal liver reaction after SBRT and can be misinterpreted with tumor progression. In a series of 26 HCC patients treated with SBRT, Price et al ${ }^{[35]}$ found that this phenomenon can even last 6 months post-SBRT. He suggested that nonenhancement on imaging, a surrogate for ablation, maybe a more useful indicator than size reduction in evaluating HCC response to SBRT in the first 6 to 12 months ${ }^{[36]}$. Sanuki-Fujimoto et al. ${ }^{[36]}$ also noted in their study that the CT appearances of the normal liver seen in reaction to the treatment of an HCC by SBRT were therefore related to background liver function and should not be misread as recurrence of HCC. 
In conclusion, Via this case report and review of literature, we suppose that bridging therapy combining SBRT and TACE may be most beneficial over TACE alone in HCC patients with a high risk of dropout from WL for LT. It is necessary to perform a randomized trial to provide more evidence to guide the treatment options for these patients.

\section{DECLARATIONS}

\section{Acknowledgments}

We acknowledge our patient for providing informed consent for this case report.

\section{Authors' contributions}

Made substantial contributions to conception and design of the case report and performed data analysis and interpretation: Bieu BQ

Performed data acquisition, as well as providing administrative, technical, and material support: Chau ND, Kien NX, Thanh LV, Quang VV, Ky TD, Thinh NT, Bang MH

Read and approved the final manuscript: Bieu BQ, Chau ND, Kien NX, Thanh LV, Quang VV, Ky TD, Thinh NT, Bang MH

\section{Availability of data and materials}

Not applicable.

\section{Financial support and sponsorship}

None.

\section{Conflicts of interest}

All authors declared that there are no conflicts of interest.

\section{Ethical approval and consent to participate}

Obtain the informed consent from patient.

\section{Consent for publication}

Not applicable.

\section{Copyright}

(c) The Author(s) 2020.

\section{REFERENCES}

1. IARC. Cancer Today. Available from: http://gco.iarc.fr/today/. [Last accessed on 24 Jul 2020]

2. Coletta M, Nicolini D, Benedetti Cacciaguerra A, Mazzocato S, Rossi R, et al. Bridging patients with hepatocellular cancer waiting for liver transplant: all the patients are the same? Transl Gastroenterol Hepatol 2017;2:78.

3. Cescon M, Cucchetti A, Ravaioli M, Pinna AD. Hepatocellular carcinoma locoregional therapies for patients in the waiting list. Impact on transplantability and recurrence rate. J Hepatol 2013;58:609-18.

4. Washburn K, Edwards E, Harper A, Freeman R. Hepatocellular carcinoma patients are advantaged in the current liver transplant allocation system. Am J Transplant 2010;10:1643-8.

5. Yao FY, Bass NM, Nikolai B, Davern TJ, Kerlan R, et al. Liver transplantation for hepatocellular carcinoma: analysis of survival according to the intention-to-treat principle and dropout from the waiting list. Liver Transpl 2002;8:873-83.

6. Yao FY, Bass NM, Nikolai B, Merriman R, Davern TJ, et al. A follow-up analysis of the pattern and predictors of dropout from the waiting list for liver transplantation in patients with hepatocellular carcinoma: implications for the current organ allocation policy. Liver Transpl 2003;9:684-92.

7. Millonig G, Graziadei IW, Freund MC, Jaschke W, Stadlmann S, et al. Response to preoperative chemoembolization correlates with outcome after liver transplantation in patients with hepatocellular carcinoma. Liver Transpl 2007;13:272-9.

8. Otto G, Herber S, Heise M, Lohse AW, Mönch C, et al. Response to transarterial chemoembolization as a biological selection criterion for 
liver transplantation in hepatocellular carcinoma. Liver Transpl 2006;12:1260-7.

9. De Luna W, Sze DY, Ahmed A, Ha BY, Ayoub W, et al. Transarterial chemoinfusion for hepatocellular carcinoma as downstaging therapy and a bridge toward liver transplantation. Am J Transplant 2009;9:1158-68.

10. Katz AW, Chawla S, Qu Z, Kashyap R, Milano MT, et al. Stereotactic hypofractionated radiation therapy as a bridge to transplantation for hepatocellular carcinoma: clinical outcome and pathologic correlation. Int J Radiat Oncol Biol Phys 2012;83:895-900.

11. O'Connor JK, Trotter J, Davis GL, Dempster J, Klintmalm GB, et al. Long-term outcomes of stereotactic body radiation therapy in the treatment of hepatocellular cancer as a bridge to transplantation. Liver Transpl 2012;18:949-54.

12. Sapisochin G, Barry A, Doherty M, Fischer S, Goldaracena N, et al. Stereotactic body radiotherapy vs. TACE or RFA as a bridge to transplant in patients with hepatocellular carcinoma. An intention-to-treat analysis. J Hepatol 2017;67:92-9.

13. Paik EK, Kim MS, Jang WI, Seo YS, Cho CK, et al. Benefits of stereotactic ablative radiotherapy combined with incomplete transcatheter arterial chemoembolization in hepatocellular carcinoma. Radiat Oncol 2016;11:22.

14. Honda Y, Kimura T, Aikata H, Kobayashi T, Fukuhara T, et al. Stereotactic body radiation therapy combined with transcatheter arterial chemoembolization for small hepatocellular carcinoma. J Gastroenterol Hepatol 2013;28:530-6.

15. Cho JY, Paik YH, Park HC, Yu JI, Sohn W, et al. The feasibility of combined transcatheter arterial chemoembolization and radiotherapy for advanced hepatocellular carcinoma. Liver Int 2014;34:795-801.

16. Kudo M, Ikeda M, Ueshima K, Sakamoto M, Shiina S, et al. Response evaluation criteria in cancer of the liver version 5 (RECICL 2019 revised version). Hepatol Res 2019;49:981-9.

17. Benedict SH, Yenice KM, Followill D, Galvin JM, Hinson W, et al. Stereotactic body radiation therapy: the report of AAPM Task Group 101. Med Phys 2010;37:4078-101.

18. Allard MA, Sebagh M, Ruiz A, Guettier C, Paule B, et al. Does pathological response after transarterial chemoembolization for hepatocellular carcinoma in cirrhotic patients with cirrhosis predict outcome after liver resection or transplantation? J Hepatol 2015;63:83-92.

19. Decaens T, Roudot-Thoraval F, Bresson-Hadni S, Meyer C, Gugenheim J, et al. Impact of pretransplantation transarterial chemoembolization on survival and recurrence after liver transplantation for hepatocellular carcinoma. Liver Transpl 2005;11:767-75.

20. She WH, Cheung TT. Bridging and downstaging therapy in patients suffering from hepatocellular carcinoma waiting on the list of liver transplantation. Transl Gastroenterol Hepatol 2016;1:34.

21. Choi SH, Seong J. Stereotactic body radiotherapy: does it have a role in management of hepatocellular carcinoma? Yonsei Med J 2018;59:912-22.

22. Blomgren H, Lax I, Näslund I, Svanström R. Stereotactic high dose fraction radiation therapy of extracranial tumors using an accelerator. Clinical experience of the first thirty-one patients. Acta Oncol 1995;34:861-70.

23. Kollmann D, Selzner N, Selzner M. Bridging to liver transplantation in HCC patients. Langenbecks Arch Surg 2017;402:863-71.

24. Sandroussi C, Dawson LA, Lee M, Guindi M, Fischer S, et al. Radiotherapy as a bridge to liver transplantation for hepatocellular carcinoma. Transpl Int 2010;23:299-306.

25. Eriguchi T, Takeda A, Sanuki N, Oku Y, Aoki Y, et al. Acceptable toxicity after stereotactic body radiation therapy for liver tumors adjacent to the central biliary system. Int J Radiat Oncol Biol Phys 2013;85:1006-11.

26. Moore A, Cohen-Naftaly M, Tobar A, Kundel Y, Benjaminov O, et al. Stereotactic body radiation therapy (SBRT) for definitive treatment and as a bridge to liver transplantation in early stage inoperable Hepatocellular carcinoma. Radiat Oncol 2017;12:163.

27. Gresswell S, Tobillo R, Hasan S, Uemura T, Machado L, et al. Stereotactic body radiotherapy used as a bridge to liver transplant in patients with hepatocellular carcinoma and Child-Pugh score $\geq 8$ cirrhosis. J Radiosurg SBRT. 2018;5:261-7.

28. Belghiti J, Carr BI, Greig PD, Lencioni R, Poon RT. Treatment before liver transplantation for HCC. Ann Surg Oncol 2008;15:993-1000.

29. European Association For The Study Of The Liver., European Organisation For Research And Treatment Of Cancer. EASL-EORTC clinical practice guidelines: management of hepatocellular carcinoma. J Hepatol 2012;56:908-43.

30. Golfieri R, Cappelli A, Cucchetti A, Piscaglia F, Carpenzano M, et al. Efficacy of selective transarterial chemoembolization in inducing tumor necrosis in small $(<5 \mathrm{~cm})$ hepatocellular carcinomas. Hepatology 2011;53:1580-9.

31. ClinicalTrials.gov. Stereotactic Body Radiation Therapy (SBRT) Versus Trans-Arterial Chemoembolization (TACE) as Bridge to Liver Transplant (SBRTvsTACE). Available from: https://clinicaltrials.gov/ct2/show/NCT02182687. [Last accessed on 24 Jul 2020].

32. ClinicalTrials.gov. Stereotactic Body Radiation Therapy (SBRT) vs Trans-Arterial Chemoembolization (TACE) as Bridge to Transplant (SBRTvsTACE). Available from: https://clinicaltrials.gov/ct2/show/NCT03960008. [Last accessed on 24 Jul 2020].

33. Jacob R, Turley F, Redden DT, Saddekni S, Aal AK, et al. Adjuvant stereotactic body radiotherapy following transarterial chemoembolization in patients with non-resectable hepatocellular carcinoma tumours of $\geq 3 \mathrm{~cm}$. HPB (Oxford) 2015;17:140-9.

34. Buckstein M, Kim E, Facciuto M, Sung M, Taouli B, et al. Phase II trial using combination of TACE and SBRT for unresectable single large HCC: interim report. Int J Radiat Oncol Biol Phys 2019;105:E214-5.

35. Price TR, Perkins SM, Sandrasegaran K, Henderson MA, Maluccio MA, et al. Evaluation of response after stereotactic body radiotherapy for hepatocellular carcinoma. Cancer 2012;118:3191-8.

36. Sanuki-Fujimoto N, Takeda A, Ohashi T, Kunieda E, Iwabuchi S, et al. CT evaluations of focal liver reactions following stereotactic body radiotherapy for small hepatocellular carcinoma with cirrhosis: relationship between imaging appearance and baseline liver function. Br J Radiol 2010;83:1063-71. 